\title{
A simple proof of exponential decay of subcritical contact processes
}

\author{
Jan M. Swart *
}

August 12, 2021

\begin{abstract}
This paper gives a new, simple proof of the known fact that for contact processes on general lattices, in the subcritical regime the expected number of infected sites decays exponentially fast as time tends to infinity. The proof also yields an explicit bound on the survival probability below the critical recovery rate, which shows that the critical exponent associated with this function is bounded from below by its mean-field value. The main idea of the proof is that if the expected number of infected sites decays slower than exponentially, then this implies the existence of a harmonic function that can be used to show that the process survives for any lower value of the recovery rate.
\end{abstract}

MSC 2010. Primary: 82C22. Secondary: 60K35, 82B $43,82 \mathrm{C} 26$.

Keywords. Subcritical contact process, sharpness of the phase transition, eigenmeasure. Acknowledgement. Work sponsored by grant 16-15238S of the Czech Science Foundation (GA $\mathrm{CR}$ ).

\section{Introduction and results}

A contact process is a Markov process $\eta=\left(\eta_{t}\right)_{t \geq 0}$ taking values in the subsets of a countable set $\Lambda$, with the following description. If $i \in \eta_{t}$, then we say that the site $i$ is infected at time $t$; otherwise it is healthy. Infected sites $i$ infect healthy sites $j$ with infection rate $a(i, j) \geq 0$, and infected sites become healthy with recovery rate $\delta \geq 0$. The formal generator of the process is given by

$$
\begin{aligned}
G f(A):= & \sum_{i, j \in \Lambda} a(i, j) 1_{\{i \in A\}} 1_{\{j \notin A\}}\{f(A \cup\{j\})-f(A)\} \\
& +\delta \sum_{i \in \Lambda} 1_{\{i \in A\}}\{f(A \backslash\{i\})-f(A)\} .
\end{aligned}
$$

In the classical set-up, $\Lambda=\mathbb{Z}^{d}$ and the infection rates are symmetric and translation-invariant, but other lattices such as regular trees have also been considered. We refer to Lig85, Lig99 as a general reference.

In what follows, we will need processes that are translation-invariant in some sense. A simple way to formalize this, which includes many classical examples such as processes on $\mathbb{Z}^{d}$ and regular trees, is to assume that $\Lambda$ is a group with group action $(i, j) \mapsto i j$, inverse operation $i \mapsto i^{-1}$, and unit element 0 (also refered to as the origin). We then assume that

${ }^{*}$ Institute of Information Theory and Automation of the ASCR (ÚTIA), Pod vodárenskou věží 4, 18208 Praha 8, Czech Republic; swart@utia.cas.cz 
the infection rates $a: \Lambda \times \Lambda \rightarrow[0, \infty)$ satisfy $a(i, i)=0(i \in \Lambda)$ and

$$
\begin{aligned}
& \text { (i) } a(i, j)=a(k i, k j) \quad(i, j, k \in \Lambda), \\
& \text { (ii) }|a|:=\sum_{i \in \Lambda} a(0, i)<\infty .
\end{aligned}
$$

Here (i) says that the infection rates are translation invariant (w.r.t. to the left action of the group on itself), while (ii) guarantees that the process is well-defined [Lig85, Thm I.3.9]. In general, we do not assume that the infection rates are symmetric, i.e., we allow for the case that $a \neq a^{\dagger}$ where we define reversed infection rates as $a^{\dagger}(i, j):=a(j, i)$. Using notation as in Swa09, SS14, we call the process with generator in (1.1) the $(\Lambda, a, \delta)$-contact process.

It is well-known [Lig85, Thm VI.1.7] that the $(\Lambda, a, \delta)$-contact process $\eta$ and the $\left(\Lambda, a^{\dagger}, \delta\right)$ contact process $\eta^{\dagger}$ are dual in the sense that

$$
\mathbb{P}\left[\eta_{t}^{A} \cap B \neq \emptyset\right]=\mathbb{P}\left[A \cap \eta_{t}^{\dagger B} \neq \emptyset\right] \quad(A, B \subset \Lambda, t \geq 0),
$$

where $\eta_{t}^{A}$ and $\eta_{t}^{\dagger B}$ denote the processes started in $\eta_{0}^{A}=A$ and $\eta_{0}^{\dagger B}=B$, respectively.

We say that the $(\Lambda, a, \delta)$-contact process survives if $\mathbb{P}\left[\eta_{t}^{A} \neq \emptyset \forall t \geq 0\right]>0$ for some, and hence for all finite nonempty $A$. We let

$$
\theta(\Lambda, a, \delta):=\mathbb{P}\left[\eta_{t}^{\{0\}} \neq \emptyset \forall t \geq 0\right]
$$

denote the survival probability started from a single infected site, and call

$$
\delta_{\mathrm{c}}=\delta_{\mathrm{c}}(\Lambda, a):=\sup \{\delta \geq 0: \theta(\Lambda, a, \delta)>0\}
$$

the critical recovery rate. It is known that $\delta_{\mathrm{c}}<\infty$. If $\Lambda$ is finite, then $\delta_{\mathrm{c}}=0$, but if $\Lambda$ is infinite, then it is often the case that $\delta_{\mathrm{c}}>0$. In particular, this is true if $\Lambda$ is finitely generated and $a$ satisfies a weak irreducibility condition [Swa07, Lemma 4.18]. For non-finitely generated infinite groups, irreducibility is in general not enough to guarantee $\delta_{\mathrm{c}}>0$ [AS10]. It is well-known that

$$
\mathbb{P}\left[\eta_{t}^{\Lambda} \in \cdot\right] \underset{t \rightarrow \infty}{\Longrightarrow} \bar{\nu}
$$

where $\bar{\nu}$ is an invariant law of the $(\Lambda, a, \delta)$-contact process, known as the upper invariant law. Using duality, it is not hard to prove that $\bar{\nu}=\delta_{\emptyset}$ if the dual $\left(\Lambda, a^{\dagger}, \delta\right)$-contact process dies out, while $\bar{\nu}$ is concentrated on the nonempty subsets of $\Lambda$ if the dual process survives Lig85, Thms VI.1.6 and 1.10]. In the latter case, we say that $\bar{\nu}$ is nontrivial.

It follows from subadditivity (see [Swa09, Lemma 1.1]) that for any $(\Lambda, a, \delta)$-contact process, there exists a constant $r=r(\Lambda, a, \delta)$ with $-\delta \leq r \leq|a|-\delta$ such that

$$
r=\lim _{t \rightarrow \infty} \frac{1}{t} \log \mathbb{E}\left[\left|\eta_{t}^{A}\right|\right] \quad \text { for all finite nonempty } A \subset \Lambda \text {. }
$$

We call $r$ the exponential growth rate. The following simple properties of $r$ are proved in [Swa09, Theorem 1.2]:

(i) $r(\Lambda, a, \delta)=r\left(\Lambda, a^{\dagger}, \delta\right)$,

(ii) The function $\delta \rightarrow r(\Lambda, a, \delta)$ is nonincreasing and Lipschitz continuous on $[0, \infty)$, with Lipschitz constant 1.

The main aim of the present paper is to present a new, simple proof of the following known fact.

Theorem 1 (Sharpness of the phase transition) For any $(\Lambda, a, \delta)$-contact process, one has $r(\Lambda, a, \delta)<0$ if and only if $\delta>\delta_{\mathrm{c}}$. 
We note that Theorem 1 and formula (1.8) (i) together imply that $\delta_{\mathrm{c}}(\Lambda, a)=\delta_{\mathrm{c}}\left(\Lambda, a^{\dagger}\right)$. By duality, it follows that for a $(\Lambda, a, \delta)$-contact process, the critical points for survival and nontriviality of the upper invariant law are the same, which in our present general setting is a nontrivial fact.

The well-known graphical representation of the contact process shows that it is, in its essence, a form of oriented percolation. Theorem 1 then says that in the whole subcritical regime, connection probabilities decay exponentially fast.

Historically, such statements were first proved for unoriented percolation, by Menshikov [Men86 and by Aizenman and Barsky [AB87]; both proofs can be found in Gri99. The proof of [AB87] is based on differential inequalities involving two parameters: the percolation parameter and the strength of an external field. Recently, Duminil-Copin and Tassion DT15a, DT15b have found a much simpler proof which depends on a single differential inequality and no longer requires the introduction of an external field.

For contact processes on $\mathbb{Z}^{d}$, Theorem 1 was first proved by Bezuidenhout and Grimmett [BG91, who adopted the method of [AB87 to the oriented, continuous-time setting. This has been generalized to processes on general transitive graphs in AJ07]; their arguments also carry over to general $(\Lambda, a, \delta)$-contact processes, as spelled out in the appendix of [SS14]. The proof of Duminil-Copin and Tassion [DT15a, DT15b] works for oriented percolation as well. With a bit of work, it is likely it can also be adapted to the continuous-time setting of the contact process.

The new proof of Theorem 1 presented here is quite different from the previous proofs. We will see that the assumption that $r(\Lambda, a, \delta)=0$ implies the existence of an, in general infinite, invariant measure for the $\left(\Lambda, a^{\dagger}, \delta\right)$-contact process, that gives rise to a harmonic function for the dual $(\Lambda, a, \delta)$-contact process. Lowering the recovery rate a bit turns this harmonic function into a subharmonic function that allows one to prove that the $(\Lambda, a, \delta-\varepsilon)$ contact process survives for any $\varepsilon>0$. This method cannot easily be adapted to unoriented percolation, but on the other hand there seems to be hope that it may be applied to more general interacting particle systems.

Proofs of sharpness of the phase transition using differential inequalities typically yield as a side result that the critical exponent associated with the function $\theta$ is bounded from below by its mean-field value 1; compare, e.g., BG91, formula (1.15)], DT15a, Part 1 of Thm 1.1] or [SS14, Lemma A.2]. Our proof also yields such a result and in fact leads to the following explicit bound.

Theorem 2 (Lower bound on survival probability) Let $\phi:(0,1) \rightarrow(0,1)$ be implicitly defined by

$$
\phi(\gamma):=1-e^{-\varepsilon} \quad \text { where } \quad \gamma=\frac{\varepsilon+e^{\varepsilon} \varepsilon}{2+e^{\varepsilon} \varepsilon} \quad(0<\varepsilon<2) .
$$

Then $\phi(\gamma)=\gamma-\frac{1}{2} \gamma^{2}+O\left(\gamma^{3}\right)$ as $\gamma \rightarrow 0$, and

$$
\theta\left(\Lambda, a,(1-\gamma) \delta_{\mathrm{c}}\right) \geq \phi(\gamma) \quad(0<\gamma<1) .
$$

\section{Proofs}

Let $\mathcal{P}=\mathcal{P}(\Lambda):=\{A: A \subset \Lambda\}$ denote the set of all subsets of $\Lambda$. We also set $\mathcal{P}_{+}:=\{A \in$ $\mathcal{P}: A \neq \emptyset\}, \mathcal{P}_{\text {fin }}:=\{A \in \mathcal{P}:|A|<\infty\}$, and $\mathcal{P}_{\text {fin },+}:=\mathcal{P}_{+} \cap \mathcal{P}_{\text {fin }}$, where $|A|$ denotes the cardinality of a set $A$. We equip $\mathcal{P} \cong\{0,1\}^{\Lambda}$ with the product topology, making it into a compact space. Now $\mathcal{P}_{+}$, being a punctured version of $\mathcal{P}$, is locally compact. Recall that a measure on a locally compact space is locally finite if it gives finite mass to compact sets. By [SS14, Lemma 1.1], a measure $\mu$ on $\mathcal{P}_{+}$is locally finite if and only if it satisfies one, and hence 
both of the following equivalent conditions:

$$
\text { (i) } \int \mu(\mathrm{d} A) 1_{\{i \in A\}}<\infty \forall i \in \Lambda \quad \text { (ii) } \int \mu(\mathrm{d} A) 1_{\{A \cap B \neq \emptyset\}}<\infty \forall B \in \mathcal{P}_{\text {fin },+} .
$$

For $A \subset \Lambda$ and $i \in \Lambda$, we write $i A:=\{i j: j \in A\}$, and for any $\mathcal{A} \subset \mathcal{P}$ we write $i \mathcal{A}:=\{i A: A \in \mathcal{A}\}$. We say that a measure $\mu$ on $\mathcal{P}$ is (spatially) homogeneous if it is invariant under the left action of the group, i.e., if $\mu(\mathcal{A})=\mu(i \mathcal{A})$ for each $i \in \Lambda$ and measurable $\mathcal{A} \subset \mathcal{P}$.

It is possible to evolve locally finite (but possibly infinite) starting measures according to the time evolution of a contact process, as follows. For a given $(\Lambda, a, \delta)$-contact process, we define subprobability kernels $P_{t}(t \geq 0)$ on $\mathcal{P}_{+}$by

$$
P_{t}(A, \cdot):=\left.\mathbb{P}\left[\eta_{t}^{A} \in \cdot\right]\right|_{\mathcal{P}_{+}} \quad\left(t \geq 0, A \in \mathcal{P}_{+}\right),
$$

where $\left.\right|_{\mathcal{P}_{+}}$denotes restriction to $\mathcal{P}_{+}$, and we define $P_{t}^{\dagger}$ similarly for the dual $\left(\Lambda, a^{\dagger}, \delta\right)$-contact process. For any measure $\mu$ on $\mathcal{P}_{+}$, we write

$$
\mu P_{t}:=\int \mu(\mathrm{d} A) P_{t}(A, \cdot) \quad(t \geq 0),
$$

which is the restriction to $\mathcal{P}_{+}$of the 'law' at time $t$ of the $(\Lambda, a, \delta)$-contact process started in the initial (possibly infinite) 'law' $\mu$. If $\mu$ is a homogeneous, locally finite measure on $\mathcal{P}_{+}$, then $\mu P_{t}$ is a homogeneous, locally finite measure on $\mathcal{P}_{+}$for each $t \geq 0$ (see [Swa09, Lemma 3.3] or [SS14, Lemma 2.4]).

Following [Swa09], we say that a measure $\mu$ on $\mathcal{P}_{+}$is an eigenmeasure of an $(\Lambda, a, \delta)$-contact process if $\mu$ is nonzero, locally finite, and there exists a constant $\lambda \in \mathbb{R}$ such that

$$
\mu P_{t}=e^{\lambda t} \mu \quad(t \geq 0)
$$

We call $\lambda$ the associated eigenvalue. We cite the following result from [Swa09, Prop. 1.4].

Lemma 3 (Existence of eigenmeasures) Each $(\Lambda, a, \delta)$-contact process has a homogeneous eigenmeasure $\stackrel{\circ}{\nu}$ with eigenvalue $r=r(\Lambda, a, \delta)$.

This is proved in Swa09] along the following lines. First, it is shown that the function $t \mapsto \mathbb{E}\left[\left|\eta_{t}^{\{0\}}\right|\right]$ is submultiplicative, which by Fekete's lemma implies that there exists a constant $r$ such that

$$
\mathbb{E}\left[\left|\eta_{t}^{\{0\}}\right|\right]=e^{r t+o(t)},
$$

where $o(t) \geq 0$ and $\lim _{t \rightarrow \infty} o(t) / t=0$. Next, one defines

$$
\nu_{\lambda}:=\int_{0}^{\infty} \mu P_{t} e^{-\lambda t} \mathrm{~d} t \quad(\lambda>r) \quad \text { with } \quad \mu:=\sum_{i \in \Lambda} \delta_{\{i\}} .
$$

Then $\nu_{\lambda} G=\lambda \nu_{\lambda}-\mu$ and $\int \nu_{\lambda}(\mathrm{d} A) 1_{\{i \in A\}}=\int_{0}^{\infty} \mathbb{E}\left[\left|\eta_{t}^{\{0\}}\right|\right] e^{-\lambda t} \mathrm{~d} t=: \pi_{\lambda}$. Since the function $o(t)$ in (2.5) is nonnegative, we have that $\pi_{\lambda} \uparrow \infty$ as $\lambda \downarrow r$. Using this, it can be shown that the normalized measures $\pi_{\lambda}^{-1} \nu_{\lambda}$ converge vaguely along some subsequence $\lambda_{n} \downarrow r$ to a limit $\stackrel{\circ}{\nu}$ which satisfies $\stackrel{\circ}{\nu} G=r \stackrel{\circ}{\nu}$, i.e., $\stackrel{\circ}{\nu}$ is an eigenmeasure with eigenvalue $r$.

In [Swa09, SS14], it is moreover proved that the eigenmeasure $\stackrel{\circ}{\nu}$ from Lemma 3 is in certain situations unique up to a multiplicative constant. For our present purposes, however, we only need existence.

It is well-known that if two Markov processes are dual, then invariant laws of one Markov process give rise to harmonic functions for its dual. A similar statement holds for eigenmeasures. We cite the following lemma from [Swa09, Lemma 3.5]. 
Lemma 4 (Eigenfunctions) Let $\mu$ be a homogeneous eigenmeasure of a $(\Lambda, a, \delta)$-contact process with eigenvalue $\lambda$. Then

$$
h(A):=\int \mu(\mathrm{d} B) 1_{\{A \cap B \neq \emptyset\} \quad\left(A \in \mathcal{P}_{\text {fin }}\right)}
$$

satisfies $G^{\dagger} h=\lambda h$, where $G^{\dagger}$ is defined as in (1.1) but for the dual $\left(\Lambda, a^{\dagger}, \delta\right)$-contact process.

Proof Proof of Theorem 11 Let $\delta_{\mathrm{c}}^{\prime}:=\inf \{\delta \geq 0: r(\Lambda, a, \delta)<0\}$. By (1.8) (ii) and the bounds $-\delta \leq r \leq|a|-\delta$, we have $r\left(\Lambda, a, \delta_{\mathrm{c}}^{\prime}\right)=0$ and $r(\Lambda, a, \delta)<0$ for all $\delta>\delta_{\mathrm{c}}^{\prime}$. It is easy to see that the $(\Lambda, a, \delta)$-contact process dies out when $r<0$, so to complete the proof it suffices to prove that the $(\Lambda, a, \delta)$-contact process survives for $\delta<\delta_{\mathrm{c}}^{\prime}$.

By (1.8) (i), $r\left(\Lambda, a^{\dagger}, \delta_{\mathrm{c}}^{\prime}\right)=0$, so Lemma 3 tells us that the $\left(\Lambda, a^{\dagger}, \delta_{\mathrm{c}}^{\prime}\right)$-contact process has a homogeneous eigenmeasure $\stackrel{\circ}{\dagger}^{\dagger}$ with eigenvalue 0 . Since $\stackrel{\circ}{\nu}^{\dagger}$ is nonzero, concentrated on $\mathcal{P}_{+}$, and homogeneous, we can normalize $\stackrel{\circ}{\nu}^{\dagger}$ such that $\int \stackrel{\circ}{\nu}^{\dagger}(\mathrm{d} B) 1_{\{i \in B\}}=1(i \in \Lambda)$. With this normalization, we define $h: \mathcal{P}_{\text {fin }} \rightarrow \mathbb{R}$ by

$$
h(A):=\int \stackrel{\circ}{\nu}^{\dagger}(\mathrm{d} B) 1_{\{A \cap B \neq \emptyset\} \quad\left(A \in \mathcal{P}_{\text {fin }}\right) .}
$$

Letting $G$ denote the generator of the $\left(\Lambda, a, \delta_{\mathrm{c}}^{\prime}\right)$-contact process, Lemma 4 tells us that $G h=0$, i.e., $h$ is a harmonic function.

Let $\varepsilon_{1}, \varepsilon_{2}>0$ be constants to be chosen later and let $\tilde{G}$ denote the generator of the $\left(\Lambda,\left(1+\varepsilon_{1}\right) a,\left(1-\varepsilon_{2}\right) \delta_{\mathrm{c}}^{\prime}\right)$-contact process. We use Lemma 5 in the appendix to transform $h$ into a bounded function $f_{\varepsilon}$ as in (A.2) such that $f_{\varepsilon}$ is subharmonic for $\tilde{G}$. To this aim, we check condition (A.3). Using the fact that $G h=0$, we see that

$$
\begin{aligned}
\tilde{G} h(A)= & \varepsilon_{1} \sum_{i, j \in \Lambda} a(i, j) 1_{\{i \in A\}} 1_{\{j \notin A\}}(h(A \cup\{j\})-h(A)) \\
& +\varepsilon_{2} \delta_{\mathrm{c}}^{\prime} \sum_{i \in \Lambda} 1_{\{i \in A\}}(h(A)-h(A \backslash\{i\})) .
\end{aligned}
$$

Let $\tilde{H}_{\varepsilon}$ be defined as in (A.4) but with $\tilde{G}$ instead of $G$, i.e.,

$$
\begin{aligned}
\tilde{H}_{\varepsilon} h(A)= & \left(1+\varepsilon_{1}\right) \sum_{i, j \in \Lambda} a(i, j) 1_{\{i \in A\}} 1_{\{j \notin A\}} \phi_{\varepsilon}(h(A \cup\{j\})-h(A)) \\
& +\left(1-\varepsilon_{2}\right) \delta_{\mathrm{c}}^{\prime} \sum_{i \in \Lambda} 1_{\{i \in A\}} \phi_{\varepsilon}(h(A \backslash\{i\})-h(A)) .
\end{aligned}
$$

It follows from (2.8) that $h$ is an increasing function in the sense that $A \subset A^{\prime}$ implies $h(A) \leq$ $h\left(A^{\prime}\right)$. Moreover, combining (2.8) with our normalization $\int \stackrel{\circ}{\nu}^{\dagger}(\mathrm{d} B) 1_{\{i \in B\}}=1$ we see that

$$
h(A)-h(A \backslash\{i\})=\int \stackrel{\circ}{\nu}^{\dagger}(\mathrm{d} B) 1_{\{A \cap B=\{i\}\}} \leq 1 \quad\left(i \in A \in \mathcal{P}_{\text {fin },+}\right) .
$$

Since $\phi_{\varepsilon}(0)=0, \phi_{\varepsilon}^{\prime}(0)=0$, and $\phi_{\varepsilon}^{\prime \prime}(z)=\varepsilon e^{-\varepsilon z}$, we see that

$$
\phi_{\varepsilon}(z) \leq \frac{1}{2} \varepsilon z^{2} \quad(0 \leq z \leq 1) \quad \text { and } \quad \phi_{\varepsilon}(z) \leq \frac{1}{2} \varepsilon e^{\varepsilon} z^{2} \quad(-1 \leq z \leq 0) .
$$

In view of this, using moreover that $z^{2} \leq|z|$ for $|z| \leq 1$, we can estimate

$$
\begin{aligned}
& \left(\tilde{G} h-\tilde{H}_{\varepsilon} h\right)(A) \\
& \geq\left[\varepsilon_{1}-\frac{1}{2} \varepsilon\left(1+\varepsilon_{1}\right)\right] \sum_{i, j \in \Lambda} a(i, j) 1_{\{i \in A\}} 1_{\{j \notin A\}}(h(A \cup\{j\})-h(A)) \\
& \quad+\left[\varepsilon_{2}-\frac{1}{2} \varepsilon e^{\varepsilon}\left(1-\varepsilon_{2}\right)\right] \delta_{\mathrm{c}}^{\prime} \sum_{i \in \Lambda} 1_{\{i \in A\}}(h(A)-h(A \backslash\{i\})),
\end{aligned}
$$


so condition (A.3) is satisfied and hence $\tilde{G} f_{\varepsilon} \geq 0$ when we choose $\varepsilon_{1}, \varepsilon_{2}$ in such a way that

$$
\frac{\varepsilon_{1}}{1+\varepsilon_{1}}=\frac{1}{2} \varepsilon \quad \text { and } \quad \frac{\varepsilon_{2}}{1-\varepsilon_{2}}=\frac{1}{2} \varepsilon e^{\varepsilon} .
$$

Let $\left(\tilde{\eta}_{t}\right)_{t \geq 0}$ denote the process with generator $\tilde{G}$, started in $\tilde{\eta}_{0}=\{0\}$, i.e., with a single infected site at the origin. It is easy to see that if a contact process with infection rates satisfying (1.2) is started in a finite initial state, then it stays finite for all time, so $\left(\tilde{\eta}_{t}\right)_{t \geq 0}$ is nonexplosive. Since $\tilde{G} f_{\varepsilon} \geq 0$ and since $f_{\varepsilon}$ is a bounded function, we have that $f_{\varepsilon}\left(\tilde{\eta}_{t}\right)$ is a bounded submartingale that converges to an a.s. $\operatorname{limit}_{t \rightarrow \infty} f_{\varepsilon}\left(\tilde{\eta}_{t}\right)=: F_{\infty}$ with

$$
\mathbb{E}\left[F_{\infty}\right] \geq f_{\varepsilon}(\{0\})=\frac{1}{\varepsilon}\left(1-e^{-\varepsilon}\right),
$$

where we have used that $h(\{0\})=1$ by our normalization of $\stackrel{\nu}{\dagger}^{\dagger}$. Since $f_{\varepsilon}(\emptyset)=0$ and $f_{\varepsilon} \leq \varepsilon^{-1}$, we have

$$
\mathbb{P}\left[\tilde{\eta}_{t} \neq \emptyset \forall t \geq 0\right] \geq \mathbb{P}\left[F_{\infty}>0\right] \geq \varepsilon \mathbb{E}\left[F_{\infty}\right] \geq 1-e^{-\varepsilon} .
$$

By a trivial rescaling of time, it follows that

$$
\theta\left(\Lambda, a, \frac{1-\varepsilon_{2}}{1+\varepsilon_{1}} \delta_{\mathrm{c}}^{\prime}\right)=\theta\left(\Lambda,\left(1+\varepsilon_{1}\right) a,\left(1-\varepsilon_{2}\right) \delta_{\mathrm{c}}^{\prime}\right) \geq 1-e^{-\varepsilon},
$$

where $\varepsilon_{1}, \varepsilon_{2}$ are defined in terms of $\varepsilon$ as in (2.14). Since $\varepsilon>0$ is arbitrary, this completes the proof. In particular, our argument shows that $\delta_{\mathrm{c}}^{\prime}=\delta_{\mathrm{c}}$.

Proof Proof of Theorem 2 We observe that by (2.14)

$$
\frac{1}{1+\varepsilon_{1}}=1-\frac{1}{2} \varepsilon \quad \text { and } \quad \frac{1}{1-\varepsilon_{2}}=1+\frac{1}{2} \varepsilon e^{\varepsilon} .
$$

Defining $\gamma$ as in (1.9), we have that

$$
\frac{1-\varepsilon_{2}}{1+\varepsilon_{1}}=\frac{1-\frac{1}{2} \varepsilon}{1+\frac{1}{2} \varepsilon e^{\varepsilon}}=1-\gamma .
$$

Then (2.17) says that

$$
\theta\left(\Lambda, a,(1-\gamma) \delta_{\mathrm{c}}\right) \geq 1-e^{-\varepsilon}
$$

which is (1.10). Since

$$
\gamma=\varepsilon+O\left(\varepsilon^{3}\right) \text { and } 1-e^{-\varepsilon}=\varepsilon-\frac{1}{2} \varepsilon^{2}+O\left(\varepsilon^{3}\right) \quad \text { as } \varepsilon \rightarrow 0,
$$

we see that $\phi(\gamma)=\gamma-\frac{1}{2} \gamma^{2}+O\left(\gamma^{3}\right)$ as $\gamma \rightarrow 0$.

\section{A Transformation of submartingales}

Let $\mathcal{S}$ be a countable set and let $G$ be a so-called Q-matrix on $\mathcal{S}$, i.e., $(G(x, y))_{x, y \in \mathcal{S}}$ are real constants such that $G(x, y) \geq 0$ for $x \neq y$ and $\sum_{y \in \mathcal{S}} G(x, y)=0$. For any real function $f$ on $\mathcal{S}$, we write

$$
G f(x):=\sum_{y \in \mathcal{S}} G(x, y) f(y)=\sum_{y \in \mathcal{S}} G(x, y)(f(y)-f(x)) \quad(x \in \mathcal{S}),
$$

whenever the infinite sums are well-defined. Then $G$ is the the generator of a (possibly explosive) continuous-time Markov chain $\left(X_{t}\right)_{t \geq 0}$ in $\mathcal{S}$. A function $h$ such that $G h \geq 0$ is called subharmonic. The following simple lemma says, roughly speaking, that an unbounded, nonnegative subharmonic function that has a sufficiently positive drift and not too large fluctuations can be transformed into a bounded subharmonic function. 
Lemma 5 (Transformation of submartingales) Let $h$ be a real function on $\mathcal{S}$ and let $\varepsilon>0$. Then the function

$$
f_{\varepsilon}(x):=\frac{1}{\varepsilon}\left(1-e^{-\varepsilon h(x)}\right) \quad(x \in \mathcal{S})
$$

satisfies $G f_{\varepsilon} \geq 0$ if and only if

$$
G h-H_{\varepsilon} h \geq 0
$$

where

$$
H_{\varepsilon} h(x):=\sum_{y \in \mathcal{S}} G(x, y) \phi_{\varepsilon}(h(y)-h(x)) \quad \text { with } \quad \phi_{\varepsilon}(z):=\varepsilon^{-1}\left(e^{-\varepsilon z}-1+\varepsilon z\right) .
$$

Proof Let $g_{\varepsilon}(z):=\varepsilon^{-1}\left(1-e^{-\varepsilon z}\right)(z \in \mathbb{R})$. Then, for any $z, z_{0} \in \mathbb{R}$,

$$
g_{\varepsilon}(z)=g_{\varepsilon}\left(z_{0}\right)+\left\{\left(z-z_{0}\right)-\phi_{\varepsilon}\left(z-z_{0}\right)\right\} e^{-\varepsilon z_{0}} \text {. }
$$

It follows that

$$
\begin{aligned}
G f_{\varepsilon}(x) & =\sum_{y \in \mathcal{S}} G(x, y)\left\{g_{\varepsilon}(h(y))-g_{\varepsilon}(h(x))\right\} \\
& =e^{-\varepsilon h(x)} \sum_{y \in \mathcal{S}} G(x, y)\left\{(h(y)-h(x))-\phi_{\varepsilon}(h(y)-h(x))\right\},
\end{aligned}
$$

which is nonnegative if and only if (A.3) holds.

\section{References}

[AB87] M. Aizenman and D.J. Barsky. Sharpness of the phase transition in percolation models. Comm. Math. Phys. 108, (1987), 489-526.

[AJ07] M. Aizenman and P. Jung. On the critical behavior at the lower phase transition of the contact process. Alea 3, (2007), 301-320.

[AS10] S.R. Athreya and J.M. Swart. Survival of contact processes on the hierarchical group. Prob. Theory Relat. Fields 147(3), (2010), 529-563.

[BG91] C. Bezuidenhout and G. Grimmett. Exponential decay for subcritical contact and percolation processes. Ann. Probab. 19(3), (1991), 984-1009.

[DT15a] H. Duminil-Copin and V. Tassion. A new proof of the sharpness of the phase transition for Bernoulli percolation and the Ising model. Commun. Math. Phys. 343(2), (2016), 725-745,

[DT15b] H. Duminil-Copin and V. Tassion. A new proof of the sharpness of the phase transition for Bernoulli percolation on $\mathbb{Z}^{d}$. Preprint (2015), arXiv:1502.03051, To appear in L'Enseignement Mathématique.

[Gri99] G. Grimmett. Percolation 2nd ed., Vol. 321 Grundlehren der Mathematischen Wissenschaften. Springer-Verlag, Berlin, 1999.

[Lig85] T.M. Liggett. Interacting Particle Systems. Springer-Verlag, New York, 1985.

[Lig99] T.M. Liggett. Stochastic interacting systems: contact, voter and exclusion processes. Springer-Verlag, Berlin, 1999. 
[Men86] M.V. Menshikov. Coincidence of the critical points in percolation problems. Soviet Math. Dokl. 33, (1986), 856-859.

[SS14] A. Sturm and J.M. Swart. Subcritical contact processes seen from a typical infected site. Electron. J. Probab. 19 (2014), no. 53, 1-46.

[Swa07] J.M. Swart. Extinction versus unbounded growth. Habilitation Thesis of the University Erlangen-Nürnberg, 2007. ArXiv:math/0702095v1.

[Swa09] J.M. Swart. The contact process seen from a typical infected site. J. Theoret. Probab. 22(3), (2009), 711-740. 